\title{
The impact of supernova remnants on the ISM in Starburst galaxies
}

\author{
Kathleen Labrie ${ }^{1}$ \\ and Chris J. Pritchet ${ }^{2}$ \\ ${ }^{1}$ Gemini Observatory, 670 N. A'ohoku Place, Hilo, HI 96720, USA email: klabrie@gemini.edu \\ ${ }^{2}$ Department of Physics and Astronomy, University of Victoria, Box 3055, Victoria, BC \\ V8W 3P6, Canada email: pritchet@uvic.ca
}

\begin{abstract}
We used the near-infrared [Fe II] emission line signature of shock-heating to detect supernova remnants (SNRs) in nearby starburst galaxies. The near-IR narrow-band imaging program has led to the detection of SNR candidates in NGC 1569 and NGC 5253, both host to super-star clusters. A spatially extended component to the [Fe II] line emission dominates the integrated [Fe II] luminosity in the two galaxies. Despite the starburst environment, the [Fe II] luminosity of the individual SNRs is two orders of magnitude lower than the luminosities observed for SNRs in M82. We find that the density and the structure of the interstellar medium is a more important factor than the starburst nature of a galaxy in determining the average [Fe II] luminosity of a SNR. The observations suggest that a significant fraction of the ISM in NGC 1569 and NGC 5253 is under the influence of SNRs. This does not appear to be the case in M82 where the impact of the SNRs is limited to high density knots.
\end{abstract}

\section{Introduction}

In normal and starburst galaxies, the near-infrared [Fe II] emission arises predominantly from the partially-ionized zone behind the shock front of radiative supernova remnants (SNRs) (Mouri, Kawara \& Taniguchi 2000). Using that emission as a tracer of SNRs, themselves long-lived tracer of the supernova activity, we can obtain insight into the impact of the supernova explosions on their host galaxy.

[Fe II] $\lambda 1.644 \mu \mathrm{m}$ and $\mathrm{Pa} \beta$ narrow-band imaging of NGC 1569 and NGC 5253 was undertaken with the REDEYE Near-IR Camera at the Canada-France-Hawaii Telescope in January 1998. The morphology of the [Fe II] emission is characterized by a somewhat textured veil of emission centred on the starburst core, and sparkled with a few compact sources. This extended component dominates the integrated [Fe II] luminosity, the compact sources accounting, respectively, for only $14 \%$ and $7 \%$ of the total [Fe II] luminosity measured in NGC 1569 and NGC 5253 (Labrie 2003; Labrie \& Pritchet 2004). Of the compact sources, 10 in NGC 1569 and 7 in NGC 5253 are supernova remnant candidates.

The [Fe II] luminosity of those SNRs is similar to the luminosity of the SNRs in M33, and of the Galactic and Magellanic remnants. This contrasts with the strikingly large luminosity of the [Fe II] sources observed in M82 (Alsonso-Herrero et al. 2001), and in a few other starburst galaxies (e.g. Forbes et al. 1993; Kotilainen et al. 1996).

\section{Analysis and Results}

The [Fe II] luminosity of radiative SNRs has been found to be proportional to the electron density, $n_{e}$, measured in the line emitting (post-shock) region of a SNR: $L_{[\mathrm{Fe} \text { II] }}=$ $1.1\left(n_{e} / \mathrm{cm}^{-3}\right) \mathrm{L}_{\odot}$ (Morel, Doyon \& St-Louis 2002). If we assume a compression ratio of 30 , a typical average for SNRs, our [Fe II] luminosity measurements indicate that the 
expected pre-shock densities at the SNRs' position should be between 2 and $20 \mathrm{~cm}^{-3}$. This is much smaller than the ISM electron density ranges actually observed in NGC 1569 and NGC $5253\left(n_{e} \sim 100 \mathrm{~cm}^{-3}\right)$. This suggests that a good fraction of the ISM in NGC 1569 and NGC 5253 is being compressed by shocks.

To investigate further, this time using the extended component to the [Fe II] emission, we statistically compared artificial images of synthetic population of [Fe II]-emitting SNRs to the real data. Again, the low pre-shock density models $\left(1 \mathrm{~cm}^{-3}<n_{\text {pre-shock }}<\right.$ $2 \mathrm{~cm}^{-3}$ ) are favored, not only based on the luminosity, but also on the morphology of the emission. The spatially extended emission can only be reproduced when the ambient density is low enough to allow the SNRs to expand.

\section{Discussion and Conclusions}

From the very large [Fe II] luminosity of sources in large starburst galaxies like M82, a dichotomy between SNRs in quiescent and starburst galaxies has been suggested. Our results contradict this hypothesis: NGC 1569 and NGC 5253 are starburst galaxies, yet the luminosity of their SNRs is similar to what is observed in quiescent galaxies. Instead of a dichotomy in SNR properties in starburst versus quiescent galaxies, we rather raise the possibility of a dichotomy in the impact of the SNRs on the ISM within the starburst galaxy family.

To explain the [Fe II] luminosity of the SNRs and the morphology of the [Fe II] emission in the starburst galaxies NGC 1569 and NGC 5253, our analysis has shown that the preshock density in those galaxies must be about $1 \mathrm{~cm}^{-3}$. Yet, the measured electron density is of the order of $100 \mathrm{~cm}^{-3}$, suggesting that most of the ISM in those galaxies is under the influence of supernova shock fronts.

This contrasts with what is observed in M82. To explain the [Fe II] luminosity of the SNRs using density arguments, the post-shock electron densities at the SNRs position must be $\sim 10^{4} \mathrm{~cm}^{-3}$. Assuming a compression ratio of 30, we obtain a pre-shock density of $\sim 300 \mathrm{~cm}^{-3}$, similar to the observed electron density in M82. This suggests that the [Fe II]-emitting SNRs in M82 are preferably found in density enhanced regions and that their impact is limited to those areas.

This raises an interesting question: why is the impact of the SN activity on the ISM so different in NGC 1569 and NGC 5253 compared to M82? Given that the galaxies are to many levels quite different, a number of factors can potentially affect the intensity and the type of feedback from the SN activity.

Too few high spatial resolution studies of the [Fe II] line emission in nearby galaxies are currently available to draw firm conclusions as to whether we have evidence for two families of starburst galaxies, or if M82 is simply a rare and exceptional individual drawn from a single family.

\section{References}

Alonso-Herrero, A., Rieke, M. J., Rieke, G. H., \& Kelly, D. M. 2001, Ap\&SS, 276, 1109

Forbes, D. A., et al. 1993, ApJ, 406, L11

Kotilainen, J. K., Forbes, D. A., Moorwood, A. F. M., van der Werf, P. P., \& Ward, M. J. 1996, A\&A, 313, 771

Labrie K. 2003, PhD thesis, University of Victoria, Victoria.

Labrie, K., \& Pritchet, C. J. 2004, in prep.

Morel, T., and Doyon, R., \& St-Louis, N. 2002, MNRAS, 329, 398

Mouri, H., Kawara, K., \& Taniguchi, Y. 2000, ApJ, 528, 186 